Spezifische Abwehr wird gestärkt

\title{
Krebskranke Kinder gegen Pneumokokken impfen!
}

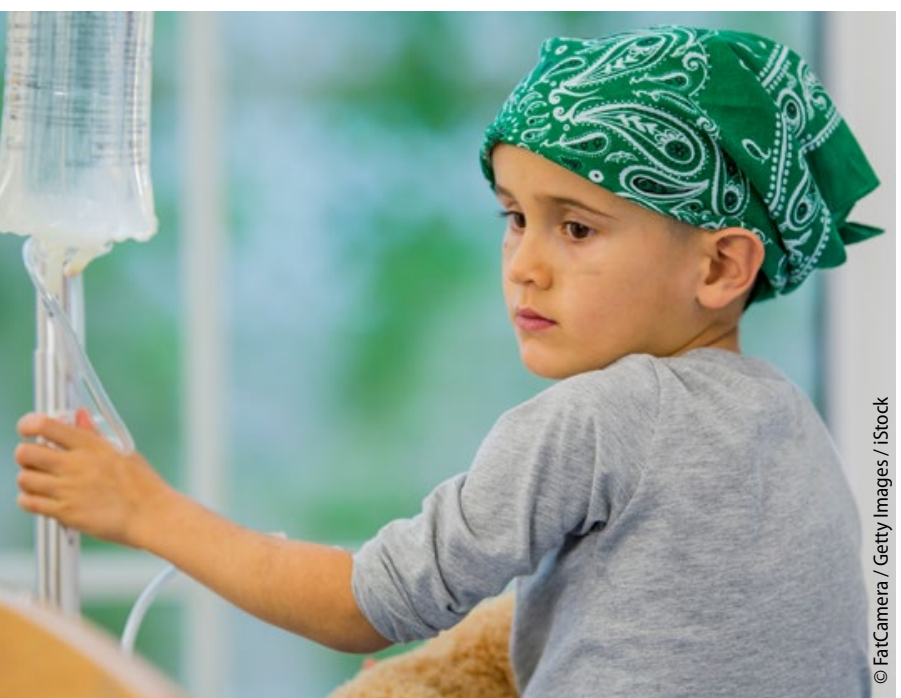

Bei krebskranken Kindern kann durch Impfung mit der 13-valenten Pneumokokkenvakzine eine schützende Immunantwort induziert werden.
Kinder, die wegen einer Krebserkrankung eine immunsuppressive Therapie erhalten, sind besonders anfällig für gefährliche Infektionen mit Streptococcus pneumoniae. Die STIKO empfiehlt daher für alle Patienten mit neoplastischer Erkrankung die Pneumokokkenimpfung.

Eine australische Studie mit 82 krebskranken Kindern bestärkt diese Empfehlung. 46 Kinder erhielten die 13-valente Konjugatvakzine (PCV13) während (A) und 36 innerhalb von zwölf Monaten nach einer Chemooder Radiotherapie (B).

Um die Wirkung der Impfung bewerten zu können, wurden die IgG-Antikör- pertiter gegen 12 der 13 Impfserotypen bestimmt. Vor der Impfung hatten die Patienten „niedrige Antikörperspiegel gegen alle Serotypen“, so die Autoren. Nach der Impfung war ein signifikanter Anstieg der Antikörperkonzentrationen festzustellen. Schützende Titer wurden zu einem höheren Prozentsatz erreicht, wenn nicht während, sondern nach der Krebstherapie geimpft wurde.

„Eine einzelne Dosis PCV13 ist bei Kindern sowohl unter einer immunsuppressiven Krebstherapie als auch in den 12 Monaten nach einer solchen Therapie immunogen", folgern die Autoren. Kinder sollten „so schnell wie möglich“ nach einer Krebsdiagnose mit PCV13 geimpft werden - unabhängig von einer früheren Exposition/Impfung. Die Studie stütze außerdem die Empfehlung für eine weitere PCV13-Impfung nach Abschluss der Krebstherapie.

Die STIKO empfiehlt dagegen für $\mathrm{Pa}-$ tienten mit Immunsuppression eine sequenzielle Impfung gegen Pneumokokken: zuerst mit PCV13 und 6-12 Monate später mit dem 23-valenten Polysaccharidimpfstoff (PPSV23).

- bs

- Hung TY et al. Cancer 2017; online 11. Juli. https://doi: 10.1002/cncr.30764

\section{Vergebliche Aufklärung}

\section{Verstärken Impfkampagnen die Impfskepsis?}

\section{Irrige Vorstellungen über die Gefahren von Impfungen lassen sich mit gängi- gen Aufklärungsstrategien offenbar nicht beseitigen - im Gegenteil.}

— Wissenschaftliche Erkenntnisse sind auch im 21. Jahrhundert erschreckend wenig durchsetzungsfähig, wenn sie im Widerspruch zu einem verbreiteten Irrglauben stehen. Das illustriert eine britischitalienische Studie, in der verschiedene Strategien getestet wurden, um Fehlurteile über Impfungen zu korrigieren.

An der Studie nahmen 120 Studenten teil, die einer von vier Informationsstrategien zugeteilt wurden: Gegenüberstellung von Impfmythen und Fakten, entweder in Textform (Gruppe 1) oder durch Tabellen und Grafiken (2) veranschaulicht, Fotos von nicht MMR-geimpften erkrankten Kindern mit entsprechenden Informationen (3) oder medizinische Informationen ohne Bezug zum Impfen (Kontrolle). Vor, direkt und sieben Tage nach Erhalt dieser Materialien wurden die Studenten nach ihrer Einstellung zum Impfen befragt.

Sowohl das Mythen-vs.-Fakten-Merkblatt als auch die Fotos erkrankter Kinder erwiesen sich als kontraproduktiv. Bei den damit konfrontierten Studenten verstärkten sie den Glauben an einen Zusammenhang zwischen Impfen und Autismus und anderen schweren Impfnebenwirkungen. Die visualisierten Impfinformationen waren zwar weniger schädlich, hatten aber auch keinerlei positive Auswirkung auf die Einstellung zu Impfungen. Sieben Tage nach dem Informationstermin waren in den Interventionsgruppen die Impfvorbehalte teilweise sogar weiter gewachsen.

Die Ergebnisse zeigen, so die Autoren, dass Fakten schnell aus dem Gedächtnis verschwinden und populäre Irrtümer zurückbleiben. Sie raten, die Wirkung von Impfkampagnen im Vorfeld besser auszutesten.

- bs

- Pluviano Set al. PLoS ONE 2017:12(7):e0181640 https://doi.org/10.1371/journal.pone.0181640 